\title{
Does the corticotropin-releasing hormone system play a role in the pathogenesis of lichen planus?
}

\author{
Bengu Cevirgen Cemil' ${ }^{1}$, Demet Yilmazer², Hatice Atas ${ }^{1}$, Fatma Pelin Cengiz ${ }^{3}$, Filiz Canpolat ${ }^{1}$, Murat Alper ${ }^{2}$, \\ Muzeyyen Gonul ${ }^{1}$
}

1Department of Dermatology, University of Health Sciences, Dışkapı Yıldııım Beyazıt Training and Research Hospital, Ankara, Turkey ${ }^{2}$ Department of Pathology, University of Health Sciences, Dışkapı Yıldırım Beyazıt Training and Research Hospital, Ankara, Turkey ${ }^{3}$ Department of Dermatovenereology, Bezmialem Vakif University, Istanbul, Turkey

\begin{abstract}
Introduction: Lichen planus (LP) is a chronic inflammatory disease, where the psychogenic factors seem to play an important role in the pathogenesis.

Aim: To determine the expression of corticotropin-releasing hormone (CRH) receptor type $1(\mathrm{CRH}-\mathrm{R} 1)$ in LP.

Material and methods: Thirty-two LP patients and 17 age/gender-matched controls were included in the study. Detailed information about the disease and body surface area (BSA) covered by the lesions was recorded. Immunohistochemically, the expression of CRH-R1 was stained in the lesional skin of patients with LP and in the control group. Results: The comparison of CRH-R1 expression showed a statistically significant difference between LP patients and the controls $(p<0.05)$. Additionally, we did not observe any correlation between BSA and staining intensity in LP patients.

Conclusions: Our study showed an increase in CRH-R1 expression in LP lesions. These results support the participation of the cutaneous CRH/CRH-R1 system in the pathogenesis of LP skin lesions.
\end{abstract}

Key words: corticotropin-releasing hormone, hypothalamo-hypophyseal system, lichen planus, receptors, skin.

\section{Introduction}

Lichen planus (LP) is an immune-mediated, chronic inflammatory disease that appears in $0.1 \%$ to $4 \%$ of the general population [1]. Although the exact etiology of LP is unclear, the evidence points to LP being a T-cell-mediated autoimmune inflammatory disorder [2]. CD8+ T lymphocytes are the major cell type seen in the dermal in flammatory infiltrate in LP; furthermore it is thought that these cells cause basal keratinocyte degeneration. There are differing opinions regarding the ethiopathogenesis of $\mathrm{LP}$, some of them correlating stress involvement with the onset and extension of the disease [3].

Stress typically results in the release of corticotropinreleasing hormone $(\mathrm{CRH})$ from the hypothalamus and regulates the hypothalamic-pituitary-adrenal (HPA) axis through activation of $\mathrm{CRH}$ receptor type 1 (CRH-R1) [4]. Recently in literature it has been reported that endocrine stress responses are not only under control of the central nervous system, but also occur in peripheral tissue, outside of the classical HPA axis [5]. The skin also contains the main components of a functional equivalent of the HPA axis [5, 6]. Cutaneous CRH is believed to regulate various functions of the skin that are important for local homeostasis [6, 7]. Many reports focus on the pro-inflammation role of $\mathrm{CRH}$, such as $\mathrm{CRH}$-induced activation of mast cells in stress-related exacerbation of cutaneous inflammatory diseases [8].

The $\mathrm{CRH}$ expressions were found in the inflamed tissues of patients with autoimmune and inflammatory diseases. For instance, inflammatory arthritis, psoriasis, vitiligo, alopecia areata, acne, seborrhea, androgenetic alopecia, age-associated skin xerosis, cutaneous lupus erythematosus, melanoma, and other skin cancers [9, 10]. To date in the literature, $\mathrm{CRH}$ expression has not been studied on the skin of LP patients.

\section{Aim}

Hereby, we have evaluated and compared the expression of the $\mathrm{CRH} / \mathrm{CRH}-\mathrm{R} 1$ system in the involved skin of LP patients and the normal skin of controls.

Address for correspondence: Bengu Cevirgen Cemil MD, Department of Dermatology, Ministry of Health Diskapi Yildirim Beyazit Education and Research Hospital, Ankara, Turkey, phone: +90 312 5962083, GSM: +90 533 6296195, fax: +90 31247295 34, e-mail: dbcemil@yahoo.com Received: 3.05.2016, accepted: 24.05.2016. 


\section{Material and methods}

\section{Patients}

Thirty-seven patients with clinically and histopathologically confirmed LP and seventeen healthy controls, ageand gender-matched with LP patients, were included in the study. The study protocol was approved by the local ethics committee, and the participants gave written consent. Subjects were selected based on the following criteria: age over eighteen years old, no history of other major physical or psychiatric illness, and no history of any regular systemic therapy or any systemic therapy for at least 3 months as well as any topical therapy for at least 1 month for LP prior to the study. Healthy controls with no history of skin or psychiatric disorders were also included in the study.

The first author of the study examined the subjects dermatologically, and the LP was confirmed by pathological biopsy prior to recruitment in the study. Detailed information about duration of the disease, clinical presentation, oral and nail manifestations, and body surface area (BSA) covered by the LP lesions were also recorded. The expression of CRH-R1 was stained in the lesional skin of patients with LP and in the control group.

\section{Immunohistochemical staining}

Immunohistochemically, the expression of CRH-R1 was investigated in the lichenoid lesions of the LP group and in the skin of the control group. For standard histology, the specimens were fixed in $10 \%$ formalin and processed for hematoxylin and eosin staining. For immunohistochemical staining, 4- $\mu \mathrm{m}$ sections were cut after which the slides were heated in oven for $60 \mathrm{~min}$ at $60^{\circ} \mathrm{C}$. For dewaxing, two Xylene washes (10 min each) followed by two $96 \%$ ethyl alcohol rinses ( 5 min each) and followed by a wash for 3 min with tap water and for 5 min by distilled water, we used the antigen-retrieval method with exposure to microwave oven heat for $20 \mathrm{~min}$ at $60^{\circ} \mathrm{C}$ and allowed them to cool down for $5 \mathrm{~min}$. Sections were immersed for 5 min in a 3\% hydrogen peroxidase solution for quenching of any endogenous peroxidases and then washed in distilled water. Non-specific protein binding was blocked for $2 \mathrm{~min}$. CRH-R1 rabbit polyclonal antibody (LifeSpan Biosciences (Catalog Number: LS-A6603), Inc, Seattle, USA) was incubated for $1 \mathrm{~h}$ with $1: 200 \mathrm{di}-$ lution protein concentration in the humidified chamber. A rabbit lgG without known specificity was used at the same concentration as the specific $A b$ in order to control non-specific staining with anti-CRHR1 Ab. After the slides were washed with two phosphate buffered saline (PBS) (5 min each), the sections were incubated for $10 \mathrm{~min}$ with biotinylated secondary antibody. Tissue sections were washed with PBS for 5 min and then incubated with peroxidase-conjugated streptavidin complex for $10 \mathrm{~min}$. The chromogenic substrate 3,3'-diaminobenzidine was used for the incubation of the sections for 5 min. Formalin-fixed and paraffin-embedded normal placenta samples were used as the positive control. In addition, formalin-fixed and paraffin-embedded breast samples were used as the negative control.

Sections were evaluated under a light microscope by a pathologist blinded to the groups. The staining results for the CRH-R1 expression of the epidermis, adnexal structures, and perivascular inflammatory infiltrate were evaluated. Positive staining cells were scored for intensity and percentage in the epidermis, adnexal structures, and perivascular inflammatory infiltrate. For staining intensity, the following four-point scale was used: grade 0 - no staining; grade 1 - mild staining; grade 2 - moderate staining; grade 3 - strong staining. The percentage of $\mathrm{CRH}-\mathrm{R} 1$ expression was defined as the percentage of positive staining cells; grade 0 for negative, grade 1 for $1-33 \%$ positive, grade 2 for $34-66 \%$ positive, and grade 3 for $67-100 \%$ positive [11].

\section{Statistical analysis}

The Statistical Package for the Social Sciences version 16.0 (SPSS, Chicago, IL, USA) was used to perform statistical analyses. Differences in the CRH-R1 expression results between the LP patients, the control group, and the LP patients with and without oral involvement were tested with the $\chi^{2}$ test. The Kruskal-Wallis test was used to compare the disease duration and BSA in the different CRH-R1 expression groups.

\section{Results}

Thirty seven patients (20 males and 17 females) were included. The mean age was $47.81 \pm 11.59$ years (range: 19 80 years). The main clinical characteristics are presented in Table 1. All healthy biopsied volunteers were found to have microscopically and pathologically normal skin.

Table 2 represents trends of cytoplasmic CRH-R1 expression, which is statistically lower in the normal control group when compared with LP patients $(p<0.05)$. CRH-R1 expression was found to be significantly increased in the epidermis, adnexal structure, and perivascular infiltrate of LP patients $(p<0.05)$. The LP group showed grade 2 or grade 3 staining of CRH-R1 on both adnexal structures and perivascular inflammatory infiltrate. The ex-

Table 1. The main clinical characteristics of all groups

\begin{tabular}{lcc}
\hline Parameter & Control & LP \\
\hline Gender (F/M) & $10 / 7$ & $20 / 17$ \\
\hline Age, mean \pm SD [years] & 46.53 & 47.81 \\
& \pm 13.91 & \pm 11.59 \\
\hline Duration of disease, mean \pm SD [month] & 29.99 \\
& \pm 56.82 \\
\hline BSA, mean \pm SD & 21.57 \\
& & \pm 17.98 \\
\hline Oral involment $(\mathrm{Y} / \mathrm{N})$ & $24 / 13$ \\
\hline
\end{tabular}


Table 2. Immunoreactivity of CRH-R1 in controls and LP patients

\begin{tabular}{|c|c|c|c|c|c|}
\hline Variable & & Lichen planus & Control & $\chi^{2}$ Test statistics & $P$-value \\
\hline \multirow[t]{4}{*}{ ISE } & 0 & 4 & 12 & & \\
\hline & + & 14 & 5 & 23.012 & $<0.001$ \\
\hline & ++ & 14 & 0 & & \\
\hline & +++ & 5 & 0 & & \\
\hline \multirow[t]{4}{*}{ ISAS } & 0 & 5 & 6 & & \\
\hline & + & 7 & 7 & 9.326 & 0.025 \\
\hline & ++ & 15 & 3 & & \\
\hline & +++ & 10 & 1 & & \\
\hline \multirow[t]{4}{*}{ ISPI } & 0 & 1 & 16 & & \\
\hline & + & 16 & 1 & 45.274 & $<0.001$ \\
\hline & ++ & 19 & 0 & & \\
\hline & +++ & 1 & 0 & & \\
\hline
\end{tabular}

ISE - intensity of staining of epidermis, ISAS - intensity of staining of the adnexal structure, ISPI - intensity of staining of perivascular inflammatory infiltrate. Intensity of staining: grade $0-$ no staining, grade $1(+)-$ mild staining, grade $2(++)$ - moderate staining, grade $3(+++)-$ strong staining. $P<0.05$ is significant.

pression of the $\mathrm{CRH}-\mathrm{R} 1$ was generally grade 0 or grade 1 on the epidermis, adnexal structures, and perivascular inflammatory infiltrate in the control group (Figures $1 \mathrm{~A}-\mathrm{H}$ ).

In patients with LP, there was no correlation between the BSA and expressions of $\mathrm{CRH}-\mathrm{R} 1$ in the epidermis,

Table 3. Correlation between the BSA and expressions of $\mathrm{CRH}-\mathrm{R} 1$

\begin{tabular}{|c|c|c|c|}
\hline Variable & & Mean \pm SD & $P$-value ${ }^{1}$ \\
\hline \multirow[t]{4}{*}{ ISE } & 0 & $29.50 \pm 11.78$ & \\
\hline & + & $22.36 \pm 16.85$ & 0.251 \\
\hline & ++ & $17.64 \pm 20.07$ & \\
\hline & +++ & $24.00 \pm 13.86$ & \\
\hline \multirow[t]{4}{*}{ ISAS } & 0 & $10.80 \pm 6.05$ & \\
\hline & + & $25.57 \pm 14.99$ & 0.255 \\
\hline & ++ & $23.87 \pm 17.81$ & \\
\hline & +++ & $20.70 \pm 20.83$ & \\
\hline \multirow[t]{4}{*}{ ISPI } & 0 & $18.00 \pm 0.00$ & \\
\hline & + & $23.38 \pm 16.10$ & 0.502 \\
\hline & ++ & $20.05 \pm 19.69$ & \\
\hline & +++ & $25.00 \pm 0.00$ & \\
\hline
\end{tabular}

ISE - intensity of staining of epidermis, ISAS - intensity of staining of the adnexal structure, ISPI - intensity of staining of perivascular inflammatory infiltrate. Intensity of staining: grade 0 , no staining; grade $1(+)$, mild staining; grade $2(++)$, moderate staining; grade $3(+++)$, strong staining. ${ }^{1} P$-values are calculated by using mean ranks of each group. the adnexal structure, and the perivascular infiltrate $(p>0.05)$ (Table 3). In addition, there was no relationship between the expression of $\mathrm{CRH}-\mathrm{R} 1$ and age, duration of the disease, gender, and oral involvement $(p>0.05)$.

\section{Discussion}

It is known that LP is a T-cell-mediated autoimmune disease; however, its etiology remains unknown [2]. It is thought that the disease is related to an immune response resulting from autoantigens due to the alteration of cell-mediated immunity triggered by endogenous or exogenous factors $[12,13]$. The autoantigens induce the activation of CD4+ $T$ and CD8+ $T$ lymphocytes as well as production of cytokines such as interleukin 1 (IL-1), IL-8, IL-10, IL-12, tumor necrosis factor $\alpha$ (TNF- $\alpha$ ), and interferon $\gamma$ $($ IFN- $\gamma)$ [12]. These cytokines increase the expression of intercellular adhesion molecules (ICAM-1) on Langerhans cells and macrophages, leading to the presentation of major histocompatibility complex antigens by means of keratinocytes. Finally, this altered immune response results in apoptosis of keratinocytes in the basal layer and may determine disease activity [13]. Different mechanisms that may also be involved in the ethiopathogenesis of the LP are mast cell degranulation and the activation of matrix metalloproteinases [14]. Furthermore, some authors believe that the chronicity of LP can be partly explained by a deficiency in the mechanisms of immunosuppression mediated by transforming growth factor $\beta$ [13]. However, the exact mechanisms that lead to the onset and chronicity of the process have not yet been fully clarified. 

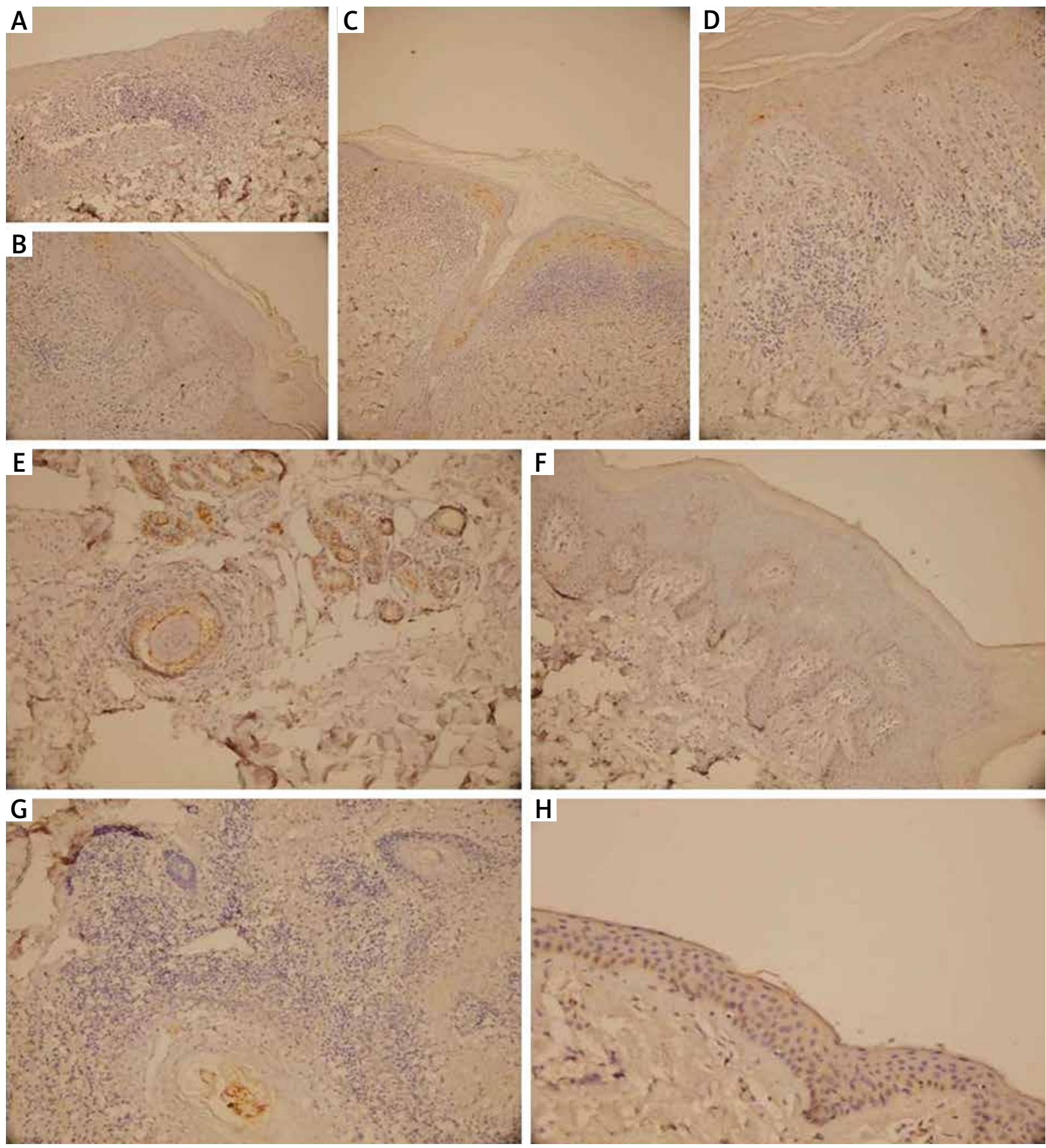

Figure 1. Sections showing immunohistochemical staining of LP patients for CRH-R1 (200x): A - epidermal intensity of staining (grade 1) and percentage (grade 2), intensity of staining and percentage of perivascular inflammatory infiltrate (grade 2), B - epidermal intensity of staining (grade 2) and percentage (grade 2), C - epidermal intensity of staining (grade 2) and percentage (grade 2), intensity of staining of perivascular inflammatory infiltrate (grade 2), D - epidermal intensity of staining (grade 3 ) and percentage (grade 1), E - intensity of staining (grade 3) and percentage (grade 3) of the adnexal structure. Sections demonstrating immunohistochemical staining of the control patients for CRH-R1 (200x), F - epidermal intensity of staining (grade 0) and percentage (grade 0), G - intensity of staining (grade 0) and percentage (grade 0) of perivascular inflammatory infiltrate and intensity of staining of the adnexal structure (grade 0 ), $\mathrm{H}$ - epidermal intensity of staining (grade 1 ) and percentage (grade 1)

The role of psychological disorders, such as depression, anxiety, and stress in the ethiopathogenesis of the $L P$ was investigated in the patients with LP in certain stud- ies. However, these studies produced controversial results. Some authors described more frequent occurrences or exacerbation of lesions during periods of greater emotional 
tension [3, 15], while others did not find any association between LP and presence of psychological alterations [16].

The skin expresses an equivalent of the prominent HPA stress axis that may act as a cutaneous defense system, operating as a coordinator and executor of local responses to stress, in addition to its normal function in preserving homeostasis [17]. Slominski et al. reported that the $\mathrm{CRH}$-based homeostatic response system represents an evolutionary preservation of an ancestral stress response mechanism since $\mathrm{CRH}$ acts as a pleiotropic cytokine in the skin [18].

In the literature, many authors have reported that $\mathrm{CRH}$ and $\mathrm{CRH}-\mathrm{Rs}$ are expressed in the skin and have certain local functions [5, 6, 19]. The biological actions of $\mathrm{CRH}$ are mediated with $\mathrm{CRH}-\mathrm{Rs}$, which may be a central element [6]. CRH-R1 is the major receptor in the epidermis and dermis [18]. Recently, research has shown that the CRH peptide family has another local function in the skin through paracrine or autocrine mechanisms [18]. For example, CRH-R1 activation stimulates diverse signal transduction pathways, which leads to the regulation of differentiation, proliferation, apoptosis, and immune activities of skin cells [7, 20]. Mitsuma et al. reported that $\mathrm{CRH}$ induces the proliferation of keratinocytes via interaction with $\mathrm{CRH}$ receptors [21], which may indicate the possible correlation of the proliferation of keratinocytes and the degree of stress. Disorganization in differentiation and proliferation of keratinocytes might result in an abnormal barrier function of the skin [5].

The $\mathrm{CRH}$ also shows biological effects such as regulation of adhesion molecules and cytokines [20, 22]. Also, recently in the literature, it has been found that human mast cells were particularly rich in both $\mathrm{CRH}$ and the structurally related peptide urocortin [23]. Furthermore, human mast cells were able to express multiple $\mathrm{CRH}$ receptor isoforms, which suggests autocrine actions of $\mathrm{CRH}$ [24]. The ability of CRH to activate mast cells may explain its proinflammatory actions and the pathophysiology of certain skin conditions, which are precipitated or exacerbated by stress, such as atopic dermatitis, eczema, psoriasis, and urticaria [25].

Previous studies revealed the functional role of the $\mathrm{CRH} / \mathrm{CRH}-\mathrm{R} 1$ system in pathological human skin conditions [7, 11]. Cemil et al. reported the expression of the $\mathrm{CRH}-\mathrm{R} 1$ in psoriatic skin and its correlation with PASI scores [11]. In the present study, for the first time we evaluated the protein expression of $\mathrm{CRH}-\mathrm{R} 1$ in the lesions of LP patients by immunohistochemistry and found the expression of CRH-R1 to be higher in these lesions than in the normal control skin. Presumably, the peripheral functions of cutaneous CRH/CRHR1 are regulated by the way of a localized circuit, and abnormal secretion of $\mathrm{CRH} / \mathrm{CRH}-\mathrm{R} 1$ in the skin due to stress disturbs the local homeostasis. Following this pathological process, abnormal differentiation, proliferation, and activation may develop in keratinocytes and mast cells [7]. So, we believe that in LP, an increased expression of $\mathrm{CRH}-\mathrm{R} 1$ may trigger and/or aggravate the disease process by interacting with keratinocytes and mast cells.

In our study, a statistically significant difference was not found between the LP patients with and without oral involvement in the expression of CRH-R1. In addition, there was no correlation between BSA and staining intensity in LP patients. Lack of serum CRH levels could be the limitation of our present study. However, following the present findings we believe that $\mathrm{CRH}-\mathrm{R} 1$ may play a role in the etiology of LP and its effects independent from the severity of the disease. New studies that evaluate both serum $\mathrm{CRH}$ level and skin $\mathrm{CRH}$ expression at the same time may show the effects of the $\mathrm{CRH}$ on the etiology of LP.

\section{Conclusions}

We demonstrated that $\mathrm{CRH}-\mathrm{R} 1$ is strongly expressed in LP skin. It may be hypothesized that a cutaneous CRH/ $\mathrm{CRH}-\mathrm{R} 1$ system might be aberrant in lesions of LP. The detuning of $\mathrm{CRH} / \mathrm{CRH}-\mathrm{R} 1$ regulation might contribute to the pathogenesis and development of LP. To our knowledge, our study is the first study to date to explore $\mathrm{CRH}$ R1 expressions in LP. Studies with a larger patient population could be the subject of future investigations and therapies.

\section{Conflict of interest}

The authors declare no conflict of interest.

\section{References}

1. Usatine RP, Tinitigan M. Diagnosis and treatment of lichen planus. Am Fam Physician 2011; 84: 53-60.

2. Ismail SB, Kumar SKS, Zain RB. Oral lichen planus and lichenoid reactions: etiopathogenesis, diagnosis, management and malignant transformation. J Oral Sci 2007; 49: 89-106.

3. Manolache L, Seceleanu-Petrescu D, Bebea V. Lichen planus patients and stressful events. J Eur Acad Dermatol Venereol 2008; 22: 437-41.

4. Chrousos GP. The hypothalamic-pituitaryadrenal axis and immune-mediated inflammation. N Engl J Med 1995; 332: 1351-62.

5. O'Kane M, Murphy EP, Kirby B. The role of corticotropinreleasing hormone in immune-mediated cutaneous inflammatory disease. Exp Dermatol 2006; 15: 143-53.

6. Slominski A, Wortsman J, Pisarchik A, et al. Cutaneous expression of corticotropin-releasing hormone $(\mathrm{CRH})$, urocortin, and CRH receptors. Faseb J 2001; 15: 1678-93.

7. Zhou C, Yu X, Cai D, et al. Role of corticotropin-releasing hormone and receptor in the pathogenesis of psoriasis. Med Hypotheses 2009; 73: 513-5.

8. Crompton R, Clifton VL, Bisits AT, et al. Corticotropin releasing hormone causes vasodilation in human skin via mast cell-dependent pathways. J Clin Endocrinol Metab 2003; 88: 5427-32. 
9. Slominski AT, Zmijewski MA, Zbytek B, et al. Key role of CRF in the skin stress response system. Endocr Rev 2013; 34: 827-84.

10. Schmitz MK, Botte DA, Sotto MN, et al. Increased corticotropin-releasing hormone $(\mathrm{CRH})$ expression in cutaneous lupus lesions. Lupus 2015; 24: 854-61.

11. Cemil BC, Canpolat F, Yilmazer D, et al. The association of PASI scores with CRH-R1 expression in patients with psoriasis. Arch Dermatol Res 2012; 3042: 127-32.

12. Payeras MR, Cherubini K, Figueiredo MA, Salum FG. Oral lichen planus: focus on etiopathogenesis. Arch Oral Biol 2013; 58: 1057-69.

13. Nico MM, Fernandes JD, Lourenço SV. Oral lichen planus. An Bras Dermatol 2011; 86: 633-41.

14. Sugerman PB, Savage NW, Walsh LJ, et al. The pathogenesis of oral lichen planus. Crit Rev Oral Biol Med 2002; 13: 350-65.

15. Rojo-Moreno JL, Bagan JV, Rojo-Moreno J, et al. Psychologic factors and oral lichen planus: a psychometric evaluation of 100 cases. Oral Surg Oral Med Oral Pathol Oral Radiol and Endod 1998; 86: 687-91.

16. Rödström PO, Jontell M, Hakeberg M, et al. Erosive oral lichen planus and salivary cortisol. J Oral Pathol Med 2001; 30: 257-63.

17. Ziegler CG, Krug AW, Zouboulis CC, Bornstein SR. Corticotropin releasing hormone and its function in the skin. Horm Metab Res 2007; 39: 106-9.

18. Slominski A, Zbytek B, Pisarchik A, et al. CRH functions as a growth factor/cytokine in the skin. J Cell Physiol 2006; 206: 780-91.

19. Zouboulis CC. The human skin as a hormone target and an endocrine gland. Hormones (Athens) 2004; 3: 9-26.

20. Quevedo ME, Slominski A, Pinto W, et al. Pleiotropic effects of corticotropin releasing hormone on normal human skin keratinocytes. In Vitro Cell Dev Biol Anim 2001; 37: 50-4.

21. Mitsuma T, Matsumoto Y, Tomita Y. Corticotropin releasing hormone stimulates proliferation of keratinocytes. Life Sci 2001; 69: 1991-8.

22. Zangeneh FZ, Fazeli A, Shooshtary FS. Psoriasis and stress psoriasis aspect of psychoneuroendocrinology. In: Psoriasis. Soung J (ed.). InTech 2012; 187-208.

23. Kempuraj D, Papadopoulou NG, Lytinas M, et al. Corticotropin-releasing hormone and its structurally related urocortin are synthesized and secreted by human mast cells. Endocrinology 2004; 145: 43-8.

24. Cao J, Zhao P, Zhao LJ, et al. Identification of functional corticotropin-releasing hormone $(\mathrm{CRH})$ receptor isoforms in human leukemic mast cells (HMC-1). Mol Biol Cell 2003; 14: L212.

25. Theoharides TC, Singh LK, Boucher W, et al. Corticotropinreleasing hormone induces skin mast cell degranulation and increased vascular permeability, a possible explanation for its proinflammatory effects. Endocrinology 1998; 139: 403-13. 